\title{
Final Program Report
}

Project Title:

Covering Period:

Date of Report:

Report \#:

Recipient:

Award Number:

Working Partners:

Cost-Sharing Partners: SiOnyx, Inc.

PI and Admin Contact: Martin Pralle

Phone: 978-922-0684x127

Fax: 978-922-0647

Email: mupralle@sionyx.com

DOE Project Team:

6/29/09- 6/30/10

July 30, 2010

SOI-0901-004

SiOnyx, Inc.

DE-EE0000581

None
Black Silicon Enhanced Thin Film Silicon Photovoltaic Devices

DOE Field Contracting Officer - $\quad$ Diana Martinez

DOE Field Project Officer - $\quad$ - (Leslie) Jim Payne

Project Engineer

Distribution Statement: Distribution Unlimited;

\section{Project Summary:}

SiOnyx has developed an enhanced thin film silicon photovoltaic device with improved efficiency. Thin film silicon solar cells suffer from low material absorption characteristics resulting in poor cell efficiencies. SiOnyx's approach leverages Black Silicon, an advanced material fabricated using ultrafast lasers. The laser treated films show dramatic enhancement in optical absorption with measured values in excess of $90 \%$ in the visible spectrum and well over $50 \%$ in the near infrared spectrum. Thin film Black Silicon solar cells demonstrate $25 \%$ higher current generation with almost no impact on open circuit voltage as compared with representative control samples. The initial prototypes demonstrated an improvement of nearly 2 percentage points in the suns Voc efficiency measurement. In addition we validated the capability to scale this processing technology to the throughputs $\left(<5 \mathrm{~min} / \mathrm{m}^{2}\right)$ required for volume production using state of the art commercially available high power industrial lasers. With these results we clearly demonstrate feasibility for the enhancement of thin film solar cells with this laser processing technique. 


\section{Project Goal:}

The goal of this Proof of Concept Feasibility Assessment program is the development of Black Silicon enhanced thin film photovoltaics that will improve the efficiency of these thin film silicon solar cells.

\section{Project Accomplishments:}

- Demonstrated 2 percentage point improvement in suns Voc efficiency verses industry standard control

- Improved current generation from 20 to $25 \mathrm{~mA} / \mathrm{cm} 2$

- Maintained Voc performance with a loss of only $2 \%$

- Validated process scale up potential using industrial lasers

- In summary we successfully demonstrated Feasibility for Black Silicon as a process enhancement in thin film silicon photovoltaics.

\section{Project Activities Summary:}

Background:

Thin-film solar cells fundamental advantage is their low materials requirements and inherent low manufacturing costs resulting $10-15 \%$ of the worldwide solar market. However, the efficiency performance of silicon based thin film cells lags behind CdTe, the dominant thin film photovoltaic technology. In order to improve the $\$ / \mathrm{W}$ ratio of thin film silicon it is necessary to increase the efficiency of these devices. Unfortunately, thinfilm silicon struggles with a trade off between efficient carrier collection and optical absorption. Thinning the junction improves drift limited carrier collection but at the expense of optical absorption.

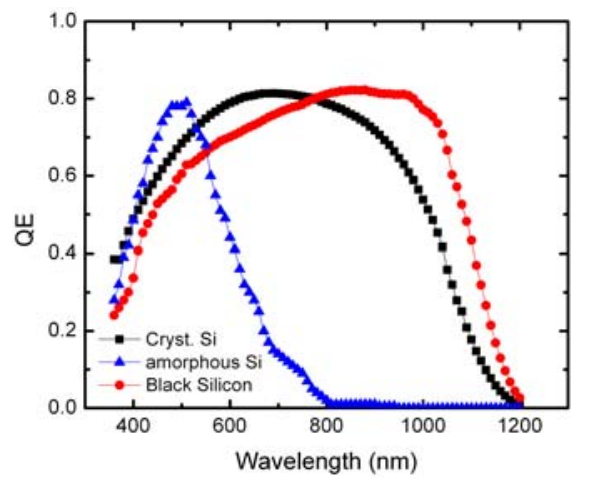

Figure 1. Quantum efficiency curves for state of the art thick crystalline Si, amorphous Si, and SiOnyx's Black

SiOnyx's Black Silicon technology, an ultrafast Silicon.

laser processing technique, enhances the absorption and quantum efficiency characteristics of silicon material, Figure 1. Integrating the spectral QE curves against the solar AM1.5 optical power spectrum we calculate $13 \%$ efficiency is possible for a thin Black Silicon photovoltaic cell, assuming equal minority carrier lifetimes. Furthermore, higher efficiencies are possible if the layer thickness can be decreased due to the enhanced absorption that Black Silicon affords. In this program we will measure the potential benefit that Black Silicon can impart in thin film silicon photovoltaics.

The Program 
During this program we developed a Black Silicon enhanced thin film photovoltaic device. The effort focused on 2 main material systems that are commonly used in the industry, amorphous silicon solar cells and microcrystalline silicon solar cells.

Although initially promising, we ran into a fundamental challenge working with hydrogenated amorphous silicon substrates. The laser process is highly energetic and during laser irradiation, pin holes and bubbles formed. After extensive investigations into the cause of these bubbles and holes we concluded that hydrogen from the hydrogenated amorphous silicon was outgassing and forming bubbles. Some of these bubbles burst causing pin holes. After multiple attempts to eliminate this effect by dehydrogenating the amorphous prior to laser processing failed we abandoned the amorphous silicon path and focused on microcrystalline films.

\section{Microcrystalline cell development}

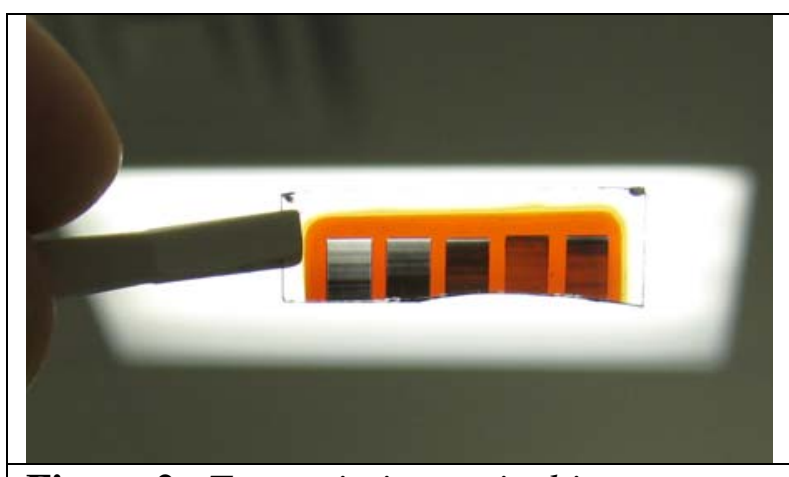

Figure 2. Transmission optical image showing Black Silicon at different laser conditions. The region on the left was processed with high power and we can observe white light transmitting through pin holes while at the optimum conditions (center) the film is mostly black.

We developed Black Silicon enhanced microcrystalline silicon cells. This work focused on production quality microcrystalline $\mathrm{p}-\mathrm{i}-\mathrm{n}$ films from an industrial supplier. The laser process did not form pin holes or bubbles in the films as was observed with the amorphous silicon material system. Therefore we were able to run laser process experiments to determine optimum laser process parameters. In Figure 2 we have a laser process experimental matrix. Optimum conditions are shown in the middle region of the sample.

Optical characterization of these films using absolute transmission and reflection measurements revealed significant increases in optical absorption with the addition of Black Silicon. Figure 3 shows a comparison of the as deposited microcrystalline film and the laser processed sample. In the visible the absorption increased to $90 \%$ and in the near IR we pushed out the absorption past $1000 \mathrm{~nm}$. This absorption improvement is essential to improve the performance of thin film silicon junctions.

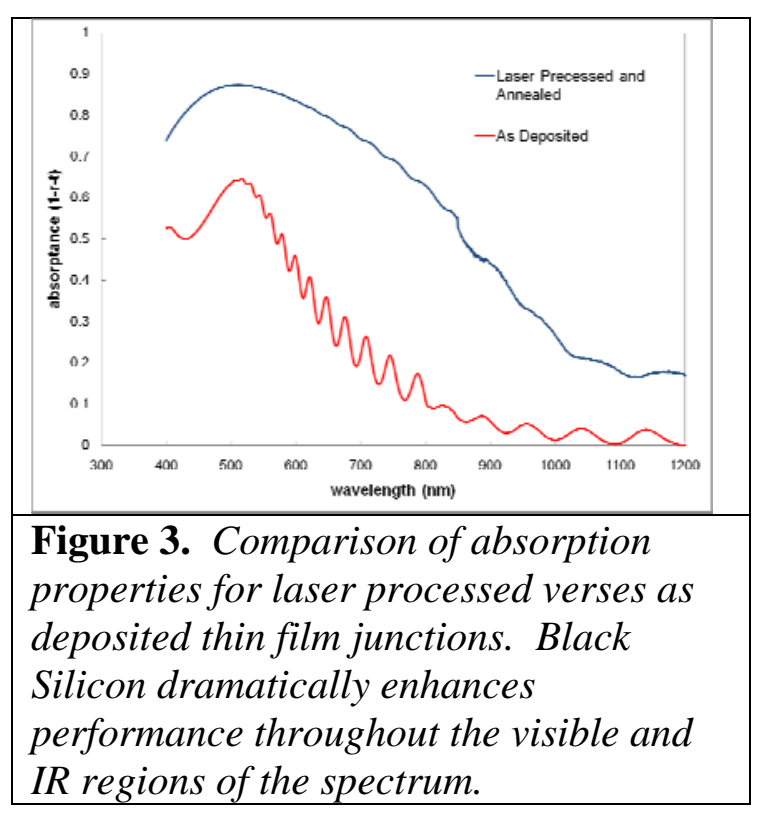

We fabricated prototype thin crystalline Black Silicon solar cells as shown in Figure 4. The low reflectivity of the Black Silicon material was maintained. 
The measured cell performance is shown in the table in Figure 5. The Black Silicon sample dramatically increased current generation with a significant impact on the open circuit voltage. The Black Silicon sample produced 25 $\mathrm{mA} / \mathrm{cm} 2$ of current as compared with 20 $\mathrm{mA} / \mathrm{cm} 2$ from the control while the Voc were comparable at 0.475 and 0.485 respectively. The Black Silicon cell's absolute efficiency was unfortunately limited by the series and shunt resistance of the cell resulting in lower performance.

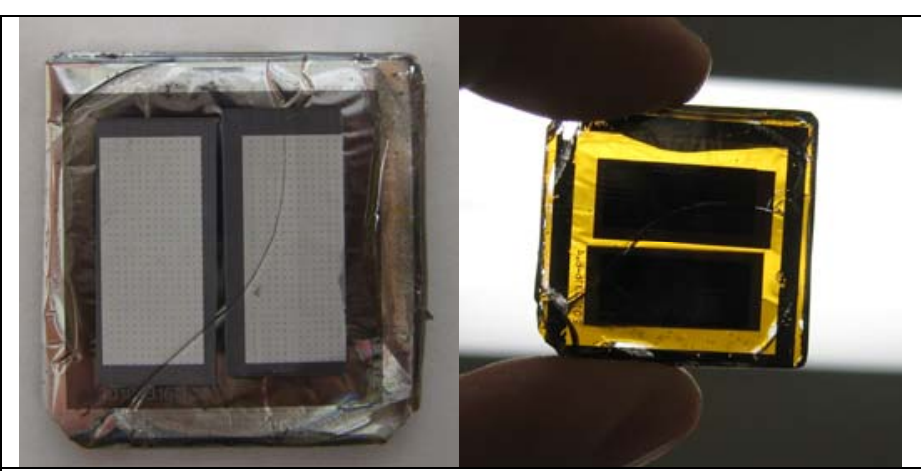

Figure 4. Picture of a thin film Black Silicon photovoltaic cell. The Black Silicon regions are the two dark rectangular regions shown on the right image. On the left is a backside view showing the metal contacts on the surface of the Black Silicon.

\begin{tabular}{|l|r|r|}
\hline & Control & SiOnyx Black Silicon \\
\hline Voc (V) & 0.485 & 0.475 \\
\hline Jsc (mA/cm2) & 20 & 25 \\
\hline Suns Voc efficiency & $7.0 \%$ & $8.9 \%$ \\
\hline
\end{tabular}

Figure 5. Final cell performance measurements. The Black Silicon enhanced cells showed a dramatic increase in current as expected from the enhanced absorption with only a marginal reduction in open circuit voltage. The Suns Voc efficiency showed almost a 2 point gain in efficiency over the control.

Quantum Efficiency was measured on thin film junctions. The quantum efficiency was demonstrated in excess of $85 \%$ and represents a major success for this program. Previous work at SiOnyx on Black Silicon demonstrated high $\mathrm{QE}$ in thick wafer devices. This thin layer QE measurement is nearly the same as the thick wafer devices and demonstrates obvious benefits for Black Silicon in thin layer devices as it clearly shows the direct conversion of the high photoabsorption into photocarriers.

Lastly, we assessed the feasibility to scale the process to

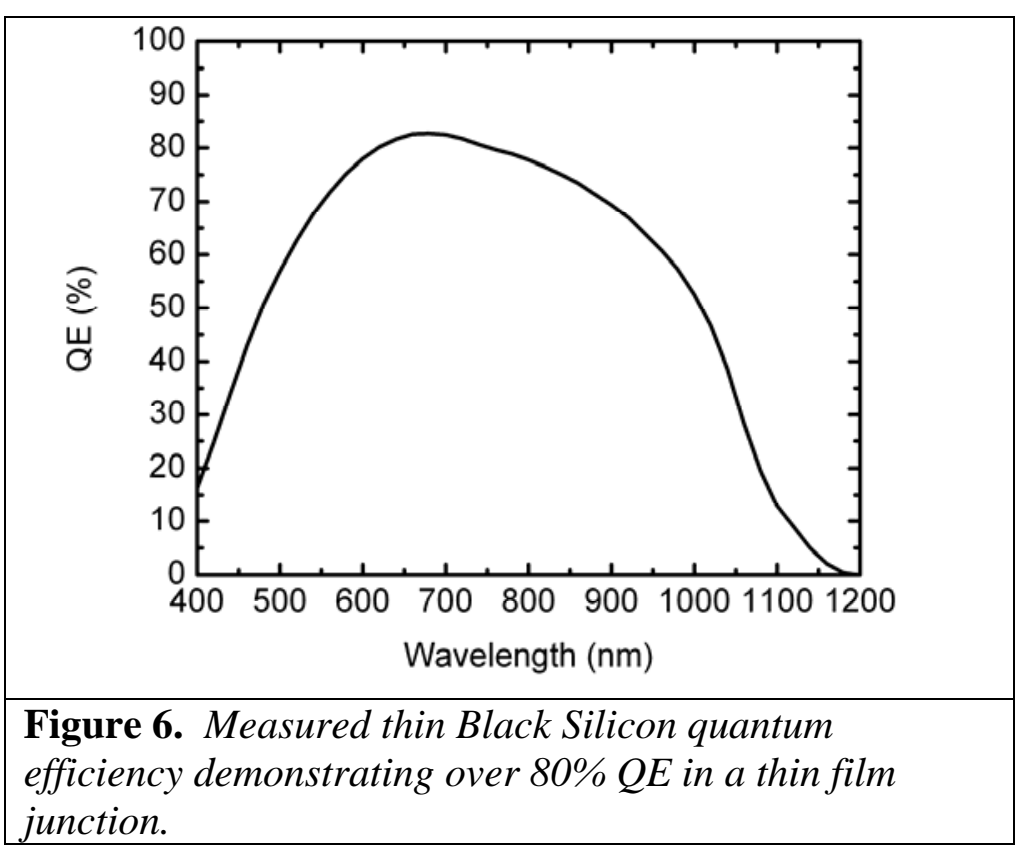
manufacturing throughputs using commercially available industrial laser from Coherent. From 
the scale up experiments we were able to determine that a manufacturing tool could process $1 \mathrm{~m}^{2}$ in under 5 minutes. This is in line with manufacturing throughput requirements.

\section{Conclusion}

In conclusion, this feasibility program was very successful in assessing the potential for Black Silicon enhancements for thin film photovoltaics. After significant research and development we are able to clearly demonstrate a significant improvement in cell performance for a thin film photovoltaic. The prototype cells showed a remarkable 25\% improvement in current collection with a marginal $2 \%$ reduction in open circuit voltage. With further engineering to improve contact and shunt resistance we clearly show a 2 percentage point improvement in efficiency potential before further optimization. This represents a very significant improvement for thin film photovoltaics and equates to a potential cost savings of $0.2 \$ / \mathrm{W}$.

\section{Technology Transfer}

This program provided the necessary feasibility to secure a larger development program to support thin film flexible photovoltaics for soldier applications funded by DARPA.

During this program a close relationship was developed between SiOnyx and the University of Delaware's Institute for Energy Conversion. This partnership continues in the above described DARPA program.

In parallel to this work SiOnyx has secured a Joint Development Agreement with Coherent Laser to develop ultrafast laser processing tools for the Solar Manufacturing industry. This strategic relationship will focus on delivering Black Silicon to the solar industry.

We anticipate publication of results in the near term. 